\title{
Switching from Exact Scheme to Nonstandard Finite Difference Scheme for Linear Delay Differential Equation
}

\author{
January 14, 2015
}

\author{
S.M. Garba ${ }^{\dagger}$, A.B. Gumel ${ }^{\ddagger}$, A.S. Hassan ${ }^{\dagger}$ and J.M-S Lubuma ${ }^{\dagger 1}$ \\ ${ }^{\dagger}$ Department of Mathematics and Applied Mathematics, University of Pretoria, Pretoria \\ 0002, South Africa. \\ ${ }^{\ddagger}$ Simon A. Levin Mathematical, Computational and Modeling Sciences Center, Arizona \\ State University, Tempe, Arizona, USA.
}

\begin{abstract}
One-dimensional models are important for developing, demonstrating and testing new methods and approaches, which can be extended to more complex systems. We design for a linear delay differential equation a reliable numerical method, which consists of two time splits as follows: (a) It is an exact scheme at the early time evolution $-\tau \leq t \leq \tau$, where $\tau$ is the discrete value of the delay; (b) Thereafter, it is a nonstandard finite difference (NSFD) scheme obtained by suitable discretizations at the backtrack points. It is shown theoretically and computationally that the NSFD scheme is dynamically consistent with respect to the asymptotic stability of the trivial equilibrium solution of the continuous model. Extension of the NSFD to nonlinear epidemiological models and its good performance are tested on a numerical example.
\end{abstract}

Keywords: Delay differential equations; exact scheme; nonstandard finite difference scheme; dynamic stability.

\footnotetext{
${ }^{1}$ Corresponding author Email: jean.lubuma@up.ac.za
} 


\section{Introduction}

Delay differential equations are extensively used in the modeling of biological systems, specifically in the epidemiology of infectious diseases $[3,7,11]$. One of the popular approaches in the study of the qualitative behaviour of such models is their linearization about the equilibria $[3,10,11,18,19]$. As a motivation, we consider the logistic delay model that arises in the modeling of communicable diseases [11], including the transmission dynamics of gonorrhea in a homosexually active population, [6]. In the latter specific case, the model is given by

$$
I^{\prime}(t)=\beta\left(1-\frac{1}{\mathcal{R}_{0}}\right) I(t)\left(1-\frac{I(t-\tau)}{N\left(1-\frac{1}{\mathcal{R}_{0}}\right)}\right),
$$

where $\beta$ is the contact rate, $\mathcal{R}_{0}$ the basic reproduction number, $N$ the total population; $\tau$, is here and after the delay in infectivity. It is evident from (1) that when $\mathcal{R}_{0} \leq 1$, the only equilibrium is the disease free equilibrium (DFE), $I^{*}=0$. However, if $\mathcal{R}_{0}>1$, in addition to the DFE, there is an endemic equilibrium (EE), $I^{* *}=N\left(1-\frac{1}{\mathcal{R}_{0}}\right)$. Linearizing (1) about the equilibria $I^{*}$ and $I^{* *}$, we have

$$
\begin{aligned}
I^{\prime}(t) & =\beta\left(1-\frac{1}{\mathcal{R}_{0}}\right) I(t) \quad \text { and } \\
X^{\prime}(t) & =-\beta\left(1-\frac{1}{\mathcal{R}_{0}}\right) X(t-\tau), \quad X(t)=I(t)-N\left(1-\frac{1}{\mathcal{R}_{0}}\right),
\end{aligned}
$$

respectively. More generally, if the spread of a disease is modeled by a system of delay differential equations, such as in $[10,18,19]$, then linearization about the equilibrium points, with the Jacobian matrix assumed to be diagonizable, leads to linearized delay differential equations of the type in (2). Thus, the general setting of this work is a linear delay differential equation (LDDE),

$$
\begin{aligned}
x^{\prime}(t) & =A x(t)+B x(t-\tau)+f(t) \quad t>0, \\
x(t) & =\phi(t) \quad t \in[-\tau, 0],
\end{aligned}
$$

where $A$ and $B$ are constants, while $f:[0,+\infty) \rightarrow \mathbb{R}$ and $\phi:[-\tau, 0] \rightarrow \mathbb{R}$ are continuous functions, with $\phi$ being the initial function.

The well-posedness of LDDE (3) can be stated as follows [9]:

Theorem 1. Under the assumptions stated above, there exists a unique continuously differentiable function $x:[-\tau,+\infty) \rightarrow \mathbb{R}$ which solves LDDE (3). The solution is represented by the Volterra integral equation

$$
\begin{aligned}
& x(t)=\phi(t), \quad t \in[-\tau, 0] \\
& x(t)=e^{A t} \phi(0)+\int_{0}^{t} e^{A(t-s)}[B x(s-\tau)+f(s)] d s, t \geq 0 .
\end{aligned}
$$


Regarding the qualitative feature of (3), we consider the homogeneous equation

$$
x^{\prime}(t)=A x(t)+B x(t-\tau)
$$

in which we assume without loss of generality that $A+B \neq 0$ so that $x=0$ is the only equilibrium point of (4). The characteristic equation of (4) is the following transcendental function of the complex argument $\lambda$ :

$$
\lambda-A-B e^{-\lambda \tau}=0 .
$$

We have the following stability result ([5], Theorem 13.8):

Theorem 2. The equilibrium $x=0$ is asymptotically stable, or equivalently, all roots of (5) have their real parts strictly less than zero if, and only if, the following two conditions hold:

(a) $A<1 / \tau$;

(b) $A<-B<\sqrt{\left(a_{1} / \tau\right)^{2}+A^{2}}$ where $a_{1}$ is the root of the equation $a=A \tan a$ with $0<a_{1}<\pi, a \in \mathbb{R}$, on the understanding that $a_{1}=\pi / 2$ if $A=0$.

In the absence of delay $(\tau=0)$ and if $f \equiv 0$, Equation LDDE (3) reduces to

$$
x^{\prime}(t)=(A+B) x(t),
$$

Equation (6) is the well-known exponential equation, which is of paramount importance from both the theoretical and numerical analysis point of view in the study of dynamical systems, without delay, of the form

$$
x^{\prime}(t)=g(x), g(0)=0 .
$$

The relevance of (6) from the constructive point of view hinges on the explicit and implicit knowledge of its exact scheme, which is [17],

$$
\frac{x_{n+1}-x_{n}}{(\exp [(A+B) \Delta t]-1) /(A+B)}=(A+B) x_{n}
$$

or

$$
\frac{x_{n+1}-x_{n}}{[1-\exp (-(A+B) \Delta t)] /(A+B)}=(A+B) x_{n+1},
$$

where $x_{n}$ denotes here and after an approximation of the solution $x(t)$ at the discrete time $t_{n}=n \Delta t, n=0,1,2, \ldots, \Delta t$ being the time step size. Most reliable nonstandard finite difference (NSFD) schemes for Equation (7) are designed on the basis of the exact scheme (8) or (9), assuming that (6) is the linearized equation of (7) about the trivial equilibrium. 
The purpose of this work is to design reliable NSFD schemes for LDDE (3). The ideal situation is to produce its exact scheme. According to Theorem 1.1 in [17], an exact scheme is readily determined once the solution of the continuous differential model is known. However, this theorem does not apply here because the second formula in Theorem 1 is an integral equation, which therefore does not give the solution explicitly. A further complication with the numerical approximation of the delay differential equation (3), already observed in the literature [4], is that the backtrack points $\left(t_{n}-\tau\right)$, $n \geq 0$, do not in general coincide with the grid points. To overcome these difficulties, we use the following time splitting strategy:

(a) We design an exact scheme of (3) for early times $t \in[-\tau, \tau]$;

(b) When $t>\tau$, we switch to the construction of a NSFD scheme.

This leads to the combined exact and $\theta$-NSFD schemes below in which $\tilde{x}_{n}$ is a suitable approximation of the solution at the backtrack points and $\psi(\Delta t)$ is a complex denominator function to be specified shortly:

$$
\frac{x_{n+1}-x_{n}}{\psi(\Delta t)}= \begin{cases}A x_{n}+\frac{1}{\psi(\Delta t)} \int_{t_{n}}^{t_{n+1}} e^{A\left(t_{n+1}-s\right)}(B \phi(s-\tau)+f(s)) d s, & \text { if } t_{n+1} \leq \tau, \\ A x_{n}+B \phi\left(t_{n}-\tau\right)+f\left(t_{n}\right), & \text { if } t_{n} \leq \tau<t_{n+1}, \\ A\left[(1-\theta) x_{n}+\theta x_{n+1}\right]+B\left[(1-\theta) \tilde{x}_{n}+\theta \tilde{x}_{n+1}\right]+f\left(t_{n}\right), & \text { if } t_{n}>\tau .\end{cases}
$$

To the authors' best knowledge, exact schemes have never been constructed for delay differential equations, while the design and implementation of NSFD schemes for such problems are not well developed.

The rest of the paper is organized as follows. In the next section, we give details on the construction of the NSFD scheme (10), while Section 3 is devoted to its dynamic consistency. Numerical simulations that support the theory are presented in Section 4. In anticipation to our future work announced in the concluding Section 5, the numerics in Section 4 are enriched with the logistic delay model on which it is shown how reliable NSFD schemes can be constructed for nonlinear epidemiological models with delay.

\section{Combined Exact and Theta-NSFD Schemes}

Let $x(t)$ be the unique solution of equation (3) given in Theorem 1. Considering the second equation at the discrete times $t_{n+1}=(n+1) \Delta t$ and $t_{n}=n \Delta t$, we have

$$
\begin{aligned}
x\left(t_{n+1}\right)-x\left(t_{n}\right) & =e^{A t_{n}}\left(e^{A \Delta t}-1\right) \phi(0)+\int_{0}^{t_{n}}\left(e^{A \Delta t}-1\right) e^{A\left(t_{n}-s\right)}[B x(s-\tau)+f(s)] d s \\
& +\int_{t_{n}}^{t_{n+1}} e^{A\left(t_{n}-s\right)} e^{A \Delta t}[B x(s-\tau)+f(s)] d s .
\end{aligned}
$$

Hence,

$$
\begin{aligned}
\frac{x\left(t_{n+1}\right)-x\left(t_{n}\right)}{\frac{\left(e^{A \Delta t}-1\right)}{A}} & =A\left[e^{A t_{n}} \phi(0)+\int_{0}^{t_{n}} e^{A\left(t_{n}-s\right)}(B x(s-\tau)+f(s)) d s\right] \\
& +\frac{A e^{A \Delta t}}{\left(e^{A \Delta t}-1\right)} \int_{t_{n}}^{t_{n+1}} e^{A\left(t_{n}-s\right)}(B x(s-\tau)+f(s)) d s .
\end{aligned}
$$


By using again the second equation in Theorem 1, Equation (12) becomes

$$
\frac{x\left(t_{n+1}\right)-x\left(t_{n}\right)}{\psi_{1}(\Delta t)}=A x\left(t_{n}\right)+\frac{1}{\psi_{1}(\Delta t)} \int_{t_{n}}^{t_{n+1}} e^{A\left(t_{n+1}-s\right)}(B x(s-\tau)+f(s)) d s,
$$

where,

$$
\psi_{1}(\Delta t)=\frac{e^{A \Delta t}-1}{A}=\Delta t+O\left(\Delta t^{2}\right)
$$

It follows, by applying the mean-value theorem to the integral in (13), that there exists $s_{n} \in\left[t_{n}, t_{n+1}\right]$ such that

$$
\begin{aligned}
\frac{1}{\psi_{1}(\Delta t)} \int_{t_{n}}^{t_{n+1}} e^{A\left(t_{n+1}-s\right)}(B x(s-\tau)+f(s)) d s & =\left[B x\left(s_{n}-\tau\right)\right. \\
& \left.+f\left(s_{n}\right)\right] \frac{1}{\psi_{1}(\Delta t)} \int_{t_{n}}^{t_{n+1}} e^{A\left(t_{n+1}-s\right)} d s \\
& =B x\left(s_{n}-r\right)+f\left(s_{n}\right) .
\end{aligned}
$$

We consider three different cases regarding the time intervals.

Case 1: Suppose that $t_{n+1} \leq \tau$. Then $s-\tau \leq t_{n+1}-\tau \leq 0$ for $s \in\left[t_{n}, t_{n+1}\right]$.

It follows from the first equation in Theorem 1 that Equation (13) reduces to the exact scheme:

$$
\frac{x\left(t_{n+1}\right)-x\left(t_{n}\right)}{\psi_{1}(\Delta t)}=A x\left(t_{n}\right)+\frac{1}{\psi_{1}(\Delta t)} \int_{t_{n}}^{t_{n+1}} e^{A\left(t_{n+1}-s\right)}(B \phi(s-\tau)+f(s)) d s .
$$

Case 2: Suppose that $t_{n+1}>\tau \geq t_{n}$. In this case, the initial condition (given by the first equation of Theorem 1) can be used to show that $x\left(t_{n}-\tau\right)=\phi\left(t_{n}-\tau\right)$.

Case 3: Suppose that $t_{n}>\tau$. In this case, the backtrack point $t_{n}-\tau$ does not necessarily coincide with a discrete time. Let $n^{*}$ be the positive integer such that $t_{n^{*}} \leq t_{n}-\tau<t_{n^{*}+1}$. We consider

$$
P(t)=x_{n^{*}+1}\left(\frac{t-t_{n^{*}}}{\Delta t}\right)-x_{n^{*}}\left(\frac{t-t_{n^{*}+1}}{\Delta t}\right)
$$

the Lagrange interpolation polynomial of degree one at the points $\left(t_{n^{*}}, x_{n^{*}}\right)$ and $\left(t_{n^{*}+1}, x_{n^{*}+1}\right)$. We approximate $x\left(t_{n}-\tau\right)$ as follows (see Figure 1 ):

$$
x\left(t_{n}-\tau\right) \simeq \tilde{x}_{n}:=P\left(t_{n}-\tau\right) .
$$

To make the approximation more explicit, we note that by construction, $n^{*}<n$ and $n^{*}$ is the integer part $\left[\frac{t_{n}-\tau}{\Delta t}\right]$ of $\frac{t_{n}-\tau}{\Delta t}$. It should further be noted that $\left[\frac{t_{n}-\tau}{\Delta t}\right]=n-m-1$, where $m \equiv m_{\Delta t}=\left[\frac{\tau}{\Delta t}\right]$. Setting $u=\frac{(m+1) \Delta t-\tau}{\Delta t} \in[0,1]$, we have,

$$
\tilde{x}_{n}=x_{n^{*}+1}\left(\frac{t_{n}-\tau-t_{n^{*}}}{\Delta t}\right)-x_{n^{*}}\left(\frac{t_{n}-\tau-t_{n^{*}+1}}{\Delta t}\right) \text {, }
$$

or,

$$
\tilde{x}_{n}=u x_{n-m}+(1-u) x_{n-m-1} .
$$

In addition to (18), we consider the following approximation of $x\left(t_{n+1}-\tau\right)$ :

$$
\tilde{x}_{n+1}=u x_{n-m+1}+(1-u) x_{n-m} .
$$


The approximation in (18) or (19) is implicit or explicit according as $m=0$ or $m>0$.

It follows from the above reasoning that the denominator function $\psi_{1}(\Delta t)$ appeared naturally. However, for our numerical scheme to capture the parameter values of the continuous model (3), we use the denominator function

$$
\psi_{2}(\Delta t)=\frac{\Delta t}{1+(Q \Delta t)^{2}}=\Delta t+O\left(\Delta t^{3}\right),
$$

where $Q \geq|A|+|B|$. Indeed, the denominator function in (20) involves the underlying parameters $A$ and $B$ instead of the function $\psi_{1}(\Delta t)$ in (14) and (13). Hence, using the weighted average of (18) and (19) through a parameter value $\theta \in[0,1]$, Equation (13) is approximated by

$$
\frac{x_{n+1}-x_{n}}{\psi_{2}(\Delta t)}=A\left[(1-\theta) x_{n}+\theta x_{n+1}\right]+B\left[(1-\theta) \tilde{x}_{n}+\theta \tilde{x}_{n+1}\right]+f\left(t_{n}\right) .
$$

It can be observed that when $\theta=0,1 / 2$ and 1 , we have the nonstandard version of the forward Euler method, trapezoidal rule and backward Euler method, respectively. To put the three cases together, we introduce the denominator function

$$
\psi(\Delta t)= \begin{cases}\psi_{1}(\Delta t), & \text { in Cases } 1 \text { and } 2 \\ \psi_{2}(\Delta t), & \text { in Case } 3\end{cases}
$$

Assume that the exact solution $x(t)$ is smooth enough and has bounded derivatives. In (18) and (19), let us replace $x_{n-m+1}, x_{n-m}$ and $x_{n-m-1}$ by the values of $x(t)$ at $t_{n-m+1}, t_{n-m}$ and $t_{n-m-1}$. Observe that

$$
x\left(t_{n+1}\right)-x\left(t_{n}\right)=\Delta t x^{\prime}\left(t_{n}\right)+\frac{(\Delta t)^{2}}{2 !} x^{\prime \prime}\left(t_{n}\right)+\cdots .
$$

Note that the local truncation error of (21) (i.e. the amount by which the solution of the continuous model fails to satisfy this numerical scheme) is defined by

$$
\begin{aligned}
T_{n+1} & =x\left(t_{n+1}\right)-x\left(t_{n}\right)-\psi_{2}(\Delta t) A\left[(1-\theta) x\left(t_{n}\right)+\theta x\left(t_{n+1}\right)\right] \\
& -\psi_{2}(\Delta t) B\left[(1-\theta) P\left(t_{n}-\tau\right)-\theta P\left(t_{n+1}-\tau\right)\right]-\psi_{2}(\Delta t) f\left(t_{n}\right) .
\end{aligned}
$$

Performing the Taylor expansion of all involved functions about $t_{n}$ and using (20), we have established the following result:

Theorem 3. The combined Exact-NSFD scheme

$$
\frac{x_{n+1}-x_{n}}{\psi(\Delta t)}= \begin{cases}A x_{n}+\frac{1}{\psi(\Delta t)} \int_{t_{n}}^{t_{n+1}} e^{A\left(t_{n+1}-s\right)}(B \phi(s-\tau)+f(s)) d s, & \text { if } t_{n+1} \leq \tau, \\ A x_{n}+B \phi\left(t_{n}-\tau\right)+f\left(t_{n}\right), & \text { if } t_{n} \leq \tau<t_{n+1}, \\ A\left[(1-\theta) x_{n}+\theta x_{n+1}\right]+B\left[(1-\theta) \tilde{x}_{n}+\theta \tilde{x}_{n+1}\right]+f\left(t_{n}\right), & \text { if } t_{n}>\tau,\end{cases}
$$

approximates the LDDE (3). This scheme is convergent, with global error being zero in the time interval $[-\tau, \tau]$. On the interval $[\tau, \infty)$, the local truncation error is in $O(\Delta t)$ if $\theta \neq 1 / 2$ and in $O\left(\Delta t^{2}\right)$ if $\theta=1 / 2$.

It should be noted that the numerical method in Theorem 3 is a NSFD scheme in the sense of $[2,17]$. Indeed, the rule on the complex denominator function of the discrete derivatives and the rule of the nonlocal approximation of right hand sides are reinforced. 
Remark 4: The NSFD theta-method was introduced in $[1,14]$ for reaction-diffusion equations and general dynamical systems. In these references, other examples of denominator functions satisfying the asymptotic relations in (14) and (20) that leads to second order convergence when $\theta=1 / 2$ are given. When $t_{n}>\tau$, computations of the NSFD scheme are performed by observing that it is a linear equation in $x_{n+1}$ which has the explicit solution

$$
x_{n+1}=\left\{\begin{array}{l}
\frac{\left[1+A(1-\theta) \psi_{2}(\Delta t)\right] x_{n}+B(1-\theta) \psi_{2}(\Delta t) \tilde{x}_{n}+B \theta \psi_{2}(\Delta t) \tilde{x}_{n+1}+\psi_{2}(\Delta t) f\left(t_{n}\right)}{1-A \theta \psi_{2}(\Delta t)}, \\
\text { if } m>0, \\
\frac{\left[1+A(1-\theta) \psi_{2}(\Delta t)+B u \theta \psi_{2}(\Delta t)\right] x_{n}+B(1-\theta) \psi_{2}(\Delta t) \tilde{x}_{n}+\psi_{2}(\Delta t) f\left(t_{n}\right)}{1-A \theta \psi_{2}(\Delta t)-B \theta(1-u) \psi_{2}(\Delta t)}, \\
\text { if } m=0 .
\end{array}\right.
$$

\section{Dynamic consistency of the NSFD scheme}

In this section, we show that the NSFD scheme preserves the stability property of the LDDE (4), as stated in Theorem 2. The conditions in this theorem regarding the parameters $A, B$ and $\tau$ are supposed to be satisfied in what follows. The NSFD scheme under consideration for (4) is given by (24) with $f\left(t_{n}\right)=0$, i.e.

$$
x_{n+1}=\left\{\begin{array}{l}
\frac{\left[1+A(1-\theta) \psi_{2}(\Delta t)\right] x_{n}+B(1-\theta) \psi_{2}(\Delta t) \tilde{x}_{n}+B \theta \psi_{2}(\Delta t) \tilde{x}_{n+1}}{1-A \theta \psi_{2}(\Delta t)}, \text { if } m>0, \\
\frac{\left[1+A(1-\theta) \psi_{2}(\Delta t)+B u \theta \psi_{2}(\Delta t)\right] x_{n}+B(1-\theta) \psi_{2}(\Delta t) \tilde{x}_{n}}{1-A \theta \psi_{2}(\Delta t)-B \theta(1-u) \psi_{2}(\Delta t)}, \text { if } m=0 .
\end{array}\right.
$$

It is clear that $x^{*}=0$ is the only fixed-point of the NSFD scheme. Thus, it preserves the unique equilibrium point $x^{*}=0$. In view of (18) and (19), the characteristic equation of the difference equation $(25)$ is

$$
H(\lambda) \equiv H_{\tau, \Delta t}(\lambda) \equiv a_{m+2} \lambda^{m+2}+a_{m+1} \lambda^{m+1}+a_{2} \lambda^{2}+a_{1} \lambda+a_{0}=0,
$$

where

$$
\begin{aligned}
& a_{m+2}=1-A \theta \psi_{2}, a_{m+1}=-\left(1+A(1-\theta) \psi_{2}\right), a_{m},=\cdots,=a_{3}=0, \\
& a_{2}=-B \theta \psi_{2} u, a_{1}=-\left[B(1-\theta) \psi_{2} u+B \theta \psi_{2}(1-u)\right], a_{0}=-B(1-\theta) \psi_{2}(1-u), \\
& \quad \text { if } m>0, \text { while } \\
& \quad a_{2}=1-A \theta \psi_{2}-B \theta(1-u) \psi_{2}, a_{1}=-\left(1+A(1-\theta) \psi_{2}+B \psi_{2} u\right), \\
& a_{0}=-B(1-\theta) \psi_{2}(1-u), \text { if } m=0 .
\end{aligned}
$$

The stability of the fixed-point using the linear delay difference equation (25) is presented by the following theorem:

Theorem 4. The fixed-point $x^{*}=0$ is asymptotically-stable for equation (25) if and only if all the roots $\lambda$ of (26) lie within the unit circle: $|\lambda|<1$

The task ahead is to check the condition $|\lambda|<1$ for every $m$. This is normally done by using the Jury's conditions [13]. However, this is a challenge because for fixed $\tau$, the degree $m$ of the polynomial in (26) increases to $\infty$ as $\Delta t$ decreases to zero. Nevertheless, we have the following partial result. 
Theorem 5. For $A+B<0$, the roots $\lambda$ of (26) satisfy the condition $|\lambda|<1$ for any $m$ whenever $B>0$ or $B<0$ with $A<B$.

Proof. Equation (26) is a special case of Volterra difference equations of convolution type investigated in [8]. According to Theorem 6.18 in this reference, the condition $|\lambda|<1$ is satisfied if

$$
\begin{aligned}
& \frac{1}{\left|1-A \theta \psi_{2}\right|}\left[\left|1+\psi_{2} A(1-\theta)\right|+\left|\psi_{2} B(1-\theta)+\psi_{2} B \theta\right|\right] \\
& =\frac{1}{\left|1-A \theta \psi_{2}\right|}\left[\left|1+\psi_{2} A(1-\theta)\right|+\left|\psi_{2} B\right|\right]<1
\end{aligned}
$$

Assume that $B>0$ (so that $A<0$ ). Then,

$$
\begin{aligned}
\frac{1}{\left|1-A \theta \psi_{2}\right|}\left[\left|1+\psi_{2} A(1-\theta)\right|+\left|\psi_{2} B\right|\right] & =\frac{1}{\left|1-A \theta \psi_{2}\right|}\left[\left|1-\psi_{2}\right| A|(1-\theta)|+\psi_{2} B\right], \\
& =\frac{1}{\left(1-A \theta \psi_{2}\right)}\left[1-\psi_{2} A \theta+\psi_{2}(A+B)\right], \\
& <\frac{\left(1-A \theta \psi_{2}\right)}{\left(1-A \theta \psi_{2}\right)}, \text { since } A+B<0, \\
& <1 .
\end{aligned}
$$

Next, we assume that $B<0$ and $A<B$. Then,

$$
\begin{aligned}
\frac{1}{\left|1-A \theta \psi_{2}\right|}\left[\left|1+\psi_{2} A(1-\theta)\right|+\left|\psi_{2} B\right|\right] & =\frac{1}{\left(1-A \theta \psi_{2}\right)}\left[\left|1-\psi_{2}\right| A|(1-\theta)|-\psi_{2} B\right], \\
& =\frac{1}{\left(1-A \theta \psi_{2}\right)}\left[1-\psi_{2}|A|(1-\theta)-\psi_{2} B\right], \\
& =\frac{1}{\left(1-A \theta \psi_{2}\right)}\left[1+\psi_{2} A-\psi_{2} A \theta-\psi_{2} A\right], \\
& \text { since }-B<-A, \\
& <\frac{\left(1-A \theta \psi_{2}\right)}{\left(1-A \theta \psi_{2}\right)} \\
& <1 .
\end{aligned}
$$

In view of Theorem 5 , the challenge raised before this result occurs actually when $A$ and $B$ satisfying the conditions in Theorem 2 are such that $B<0$ and $A>B$. Since the theorem in [8] that was used in the proof of Theorem 5 is not a necessary condition for $|\lambda|<1$ to hold, we will for the case under consideration check Theorem 4 fully for $m=0,1$, and partially for $m=2$.

The case $m=0$, i.e. $0 \leq \tau<\Delta t$

The Jury conditions for the polynomial in (26) read:

(1) $H(1)>0, H(-1)>0$.

(2) $a_{0}-a_{2}<0, a_{0}+a_{2}>0$.

By definition,

$$
\begin{aligned}
H(1) & =1-\theta \psi_{2}(A+B)+B \psi_{2} \theta u-B \psi_{2} \theta u-1-\psi_{2}(A+B)+\theta \psi_{2}(A+B), \\
& =-\psi_{2}(A+B)>0, \text { since } A+B<0 .
\end{aligned}
$$


Similarly,

$$
\begin{aligned}
& H(-1)=2+\psi_{2} A-2 \psi_{2} \theta A+2 \psi_{2} B u-\psi_{2} B, \\
& =2+(A-B) \psi_{2}-2 \psi_{2} \theta A+2 \psi_{2} B u, \\
& >2-2 \psi_{2} \theta A+2 \psi_{2} B u, \text { as }(A-B)>0, \\
& >1+2 B \psi_{2} \theta,(-A>B), \\
& >1+2 B \psi_{2} \theta \\
& >0, \text { since } \psi_{2}<\frac{1}{-B} \text { in view of the definition of } \psi_{2} \text { in }(20) \text { which implies that } \\
& \qquad \psi_{2}<\frac{1}{|A|+|B|} .
\end{aligned}
$$

From condition (2) above, we have

$$
\begin{aligned}
a_{0}-a_{2} & =-B \psi_{2}+B \psi_{2} u+B \psi_{2} \theta-B \psi_{2} u \theta-1+A \theta \psi_{2}+B \psi_{2} \theta-B \psi_{2} \theta u, \\
& <-1+B-B \psi_{2}(u-1)+B \psi_{2} \theta-B \psi_{2} \theta+A \theta \psi_{2}+B \psi_{2} \theta-B \psi_{2} \theta, \\
& <-1+A \theta \psi_{2}, \\
& <-1+B \theta \psi_{2}, \\
& <0, \text { since } B<0 .
\end{aligned}
$$

Similarly,

$$
\begin{aligned}
a_{0}+a_{2} & =-B \psi_{2}+B \psi_{2} u+B \psi_{2} \theta-B \psi_{2} u \theta+1-A \theta \psi_{2}-B \psi_{2} \theta+B \psi_{2} \theta u, \\
& =B \psi_{2}(u-1)+1-A \theta \psi_{2}, \\
& >B \psi_{2}(u-1)+1+B \theta \psi_{2}, \\
& >1+B \psi_{2}, \\
& >0 \text { by (28). }
\end{aligned}
$$

Therefore, according to Jury's stability conditions, when $m=0$, all the roots of $H(\lambda)$ lie within the unit circle. Hence, $x^{*}=0$ is asymptotically stable.

The case $m=1$, i.e. $\Delta t \leq \tau<2 \Delta t$

The Jury conditions for the polynomial in (26) read:

$$
\begin{aligned}
H(1) & >0, \\
H(-1) & <0, \\
b_{0}-b_{2} & <0, \\
b_{0}+b_{2} & <0,
\end{aligned}
$$

where,

$$
\begin{aligned}
b_{0} & =\left(B \psi_{2}-B \psi_{2} u-B \psi_{2} \theta+B \psi_{2} \theta u\right)^{2}-\left(1-A \theta \psi_{2}\right)^{2}, \\
b_{2} & =\left(B \psi_{2}-B \psi_{2} u-B \psi_{2} \theta+B \psi_{2} \theta u\right)\left(1+A \psi_{2}-A \theta \psi_{2}+B \psi_{2} \theta u\right) \\
& +\left(B \psi_{2} u-B \psi_{2} \theta u+B \psi_{2} \theta-B \psi_{2} \theta u\right)\left(1-A \theta \psi_{2}\right) .
\end{aligned}
$$

The condition $(29)_{1}$ is straightforward because $H(1)=-\psi_{2}(A+B)>0$ as $A+B<0$. 
Likewise, from condition $(29)_{2}$, we have,

$$
\begin{aligned}
H(-1) & =-2+2 \psi_{2} \theta A-\psi_{2}(A+B)-4 B \theta \psi_{2} u+2 B \psi_{2} \theta+2 B \psi_{2} u, \\
& <-2+2 A \psi_{2} \theta-\psi_{2}(A+B)-2 B \psi_{2} u+2 B \psi_{2} u-2 B \psi_{2} \theta+2 B \psi_{2} \theta, \\
& <-2+2 B \psi_{2} \theta-\psi_{2}(-B+B),(B<A,-B>A) \\
& <-2+B \psi_{2} \theta \\
& <0,(B<0) .
\end{aligned}
$$

From the expression,

$$
\begin{aligned}
b_{0}-b_{2} & =\left(B \psi_{2}-B \psi_{2} u-B \psi_{2} \theta+B \psi_{2} \theta u\right)^{2} \\
& -\left(B \psi_{2}-B \psi_{2} u-B \psi_{2} \theta+B \psi_{2} \theta u\right)\left(1+A \psi_{2}-A \theta \psi_{2}+B \psi_{2} \theta u\right) \\
& -\left(B \psi_{2} u-B \psi_{2} \theta u+B \psi_{2} \theta-B \psi_{2} \theta u\right)\left(1-A \theta \psi_{2}\right), \\
& <\left[B \psi_{2} \theta(u-1)\right]^{2}-\left[B \psi_{2} \theta(u-1)\left(1+A \psi_{2}-A \theta \psi_{2}+B \psi_{2} \theta u\right)\right], \\
& <B \psi_{2} \theta(u-1)-1-A \psi_{2}+A \theta \psi_{2}-B \psi_{2} \theta u, \\
& <B \psi_{2} \theta(u-1-u)-1-A \psi_{2}+A \theta \psi_{2}, \\
& <-B \psi_{2} \theta-1-A \psi_{2}(1-\theta), \text { if } A>0, \\
& <-B \psi_{2}-1, \\
& <0, \text { by }(28) .
\end{aligned}
$$

If $A<0$, then

$$
\begin{aligned}
b_{0}-b_{2} & <-B \psi_{2} \theta-1-A \psi_{2}(1-\theta), \\
& <-B \psi_{2}-1-A \psi_{2}, \\
& <0, \text { since } \psi_{2}<\frac{1}{-(A+B)} \text { by }(28) .
\end{aligned}
$$

Similarly, from the condition $(29)_{4}$,

$$
\begin{aligned}
b_{0}+b_{2} & =\left(B \psi_{2}-B \psi_{2} u-B \psi_{2} \theta+B \psi_{2} \theta u\right)^{2} \\
& +\left(B \psi_{2}-B \psi_{2} u-B \psi_{2} \theta+B \psi_{2} \theta u\right)\left(1+A \psi_{2}-A \theta \psi_{2}+B \psi_{2} \theta u\right) \\
& -\left(B \psi_{2} u-B \psi_{2} \theta u+B \psi_{2} \theta-B \psi_{2} \theta u\right)\left(1-A \theta \psi_{2}\right), \\
& <\left[B \psi_{2} \theta(u-1)\right]^{2}+\left[B \psi_{2} \theta(u-1)\left(1+A \psi_{2}-A \theta \psi_{2}+B \psi_{2} \theta u\right)\right], \\
& <B \psi_{2} \theta(u-1)+1+A \psi_{2}-A \theta \psi_{2}+B \psi_{2} \theta u, \\
& =B \psi_{2} \theta(2 u-1)+1+A \psi_{2}-A \theta \psi_{2}, \\
& <B \psi_{2} \theta+1+A \psi_{2}-B \theta \psi_{2}, \\
& <0, \text { since } \psi_{2}<\frac{1}{|A|} \text { by }(28) .
\end{aligned}
$$

From (29), the Jury's stability conditions are satisfied, with $m=1$, hence all the roots of $H(\lambda)$ lie within the unit circle. Therefore the equilibrium point $x^{*}=0$ is asymptotically stable. 
The case $m=2$, i.e. $2 \Delta t \leq \tau<3 \Delta t$

The Jury conditions for the polynomial in (26) read:

$$
\begin{aligned}
H(1) & >0, \\
H(-1) & >0, \\
a_{0}-a_{4} & <0, \\
a_{0}+a_{4} & >0, \\
c_{0}-c_{2} & >0, \\
c_{0}+c_{2} & >0,
\end{aligned}
$$

where,

$$
\begin{aligned}
c_{0}=b_{0}^{2}-b_{3}^{2} & =\left[\left(B \psi_{2}-B \psi_{2} u-B \psi_{2} \theta+B \psi_{2} \theta u\right)^{2}-\left(1-A \theta \psi_{2}\right)^{2}\right]^{2} \\
- & {\left[\left(B \psi_{2}-B \psi_{2} u-B \psi_{2} \theta+B \psi_{2} \theta u\right)\left(1+A \psi_{2}-A \theta \psi_{2}\right)\right.} \\
+ & \left.\left(B \psi_{2} u-B \psi_{2} \theta u+B \psi_{2} \theta-B \psi_{2} \theta u\right)\left(1-A \theta \psi_{2}\right)\right]^{2} \\
c_{2}=b_{0} b_{2}-b_{1} b_{3}=\left[\left(B \psi_{2}-B \psi_{2} u-B \psi_{2} \theta+B \psi_{2} \theta u\right)^{2}-\left(1-A \theta \psi_{2}\right)^{2}\right] & {\left.\left[\left(B \psi_{2}-B \psi_{2} u-B \psi_{2} \theta+B \psi_{2} \theta u\right)+\left(1-A \theta \psi_{2}\right)\right) B \psi_{2} \theta u\right] } \\
- & {\left[\left(B \psi_{2}-B \psi_{2} u-B \psi_{2} \theta+B \psi_{2} \theta u\right)\left(B \psi_{2} u-B \psi_{2} \theta u+B \psi_{2} \theta-B \psi_{2} \theta u\right)\right.} \\
+ & \left.\left(1-A \theta \psi_{2}\right)\left(1+A \psi_{2}-A \theta \psi_{2}\right)\right]\left[\left(B \psi_{2}-B \psi_{2} u-B \psi_{2} \theta+B \psi_{2} \theta u\right)\right. \\
& \left.\left(1+A \psi_{2}-A \theta \psi_{2}\right)+\left(B \psi_{2} u-B \psi_{2} \theta u+B \psi_{2} \theta-B \psi_{2} \theta u\right)\left(1-A \theta \psi_{2}\right)\right]
\end{aligned}
$$

The conditions $(30)_{1}$, is obtained as follows,

$$
\begin{aligned}
H(1) & =-\psi_{2}(A+B) \\
& >0, \text { as } A+B<0 .
\end{aligned}
$$

To check $(30)_{2}$, we have,

$$
\begin{aligned}
H(-1) & =2-2 A \psi_{2} \theta+A \psi_{2}-4 B \theta \psi_{2} u+2 B u \psi_{2}+2 B \theta \psi_{2} u+2 B \theta \psi_{2}-B \psi_{2}, \\
& >2+\psi_{2} A(2 \theta-1)-2 \psi_{2} B u(2 \theta-1)+B \psi_{2}(2 \theta-1), \\
& =2+\psi_{2}(2 \theta-1)(A+B)-2 \psi_{2} B u(2 \theta-1) .
\end{aligned}
$$

we distinguish two cases :

When $\theta \in[0,1 / 2]$, i.e. $(2 \theta-1) \leq 0$, we have

$$
\begin{aligned}
H(-1) & >2-2 \psi_{2} B u(2 \theta-1), \\
& >1-1 \psi_{2} B(2 \theta-1), \\
& >0, \text { since } \psi_{2}<\frac{1}{B(2 \theta-1)}, \text { by }(28) .
\end{aligned}
$$

When $\theta \in(1 / 2,1]$, i.e. $(2 \theta-1) \geq 0$, we have

$$
\begin{aligned}
H(-1) & >2+\psi_{2}(2 \theta-1)(A+B)-2 \psi_{2} B u(2 \theta-1), \\
& >1+\psi_{2}(2 \theta-1)(A+B), \\
& >0, \text { as } \psi_{2}<\frac{1}{-(A+B)(2 \theta-1)} \text { by }(28) .
\end{aligned}
$$


Condition $(30)_{3}$ and $(30)_{4}$, are obtained as follows from (28):

$$
\begin{aligned}
a_{0}-a_{4} & =-B \psi_{2}+B \psi_{2} u+B \psi_{2} \theta-B \psi_{2} u \theta-1+A \theta \psi_{2}, \\
& <-B \psi_{2}+B \psi_{2} \theta u-B \psi_{2} \theta u-1+(A+B) \theta \psi_{2}, \\
& <-1-B \psi_{2}, \\
& <0
\end{aligned}
$$

and

$$
\begin{aligned}
a_{0}+a_{4} & =-B \psi_{2}+B \psi_{2} u+B \psi_{2} \theta-B \psi_{2} u \theta+1-A \theta \psi_{2}, \\
& >1+(B-A) \psi_{2} \theta, \\
& >1+(B-A) \psi_{2}, \\
& >0 .
\end{aligned}
$$

After some computations, the quantities involved in conditions $(30)_{5}$ and $(30)_{6}$ are given by

$$
\begin{aligned}
c_{0}-c_{2} & =\left[\left(B \psi_{2}(1-\theta)(1-u)\right)^{2}-\left(1-A \theta \psi_{2}\right)^{2}\right]^{2} \\
& -\left[\left(B \psi_{2}(1-u)(1-\theta)\right)\left(1+A \psi_{2}-A \theta \psi_{2}\right)+\left(B \psi_{2} u(1-\theta)+B \psi_{2} \theta(1-u)\left(1-A \theta \psi_{2}\right)\right]^{2}\right. \\
& -\left[\left(B \psi_{2}(1-\theta)(1-u)\right)^{2}-\left(1-A \theta \psi_{2}\right)^{2}\right]\left[\left(B \psi_{2}(1-\theta)(1-u)\right) B \psi_{2} \theta u\right. \\
& \left.+\left(1-A \theta \psi_{2}\right) B \psi_{2} \theta u\right]+\left[\left(B \psi_{2}(1-\theta)(1-u)\right)\left(B \psi_{2} u(1-\theta)\right)\right. \\
& \left.+\left(1-A \theta \psi_{2}\right)\left(1+A \psi_{2}-A \theta \psi_{2}\right)\right]\left[\left(B \psi_{2}(1-\theta)(1-u)\right)\left(1+A \psi_{2}-A \theta \psi_{2}\right)\right. \\
& \left.+\left(B \psi_{2} u(1-\theta)+B \psi_{2} \theta(1-u)\right)\left(1-A \theta \psi_{2}\right)\right] .
\end{aligned}
$$

and

$$
\begin{aligned}
c_{0}+c_{2} & =\left[\left(B \psi_{2}(1-\theta)(1-u)\right)^{2}-\left(1-A \theta \psi_{2}\right)^{2}\right]^{2} \\
& -\left[\left(B \psi_{2}(1-u)(1-\theta)\right)\left(1+A \psi_{2}-A \theta \psi_{2}\right)+\left(B \psi_{2} u(1-\theta)+B \psi_{2} \theta(1-u)\left(1-A \theta \psi_{2}\right)\right]^{2}\right. \\
& +\left[\left(B \psi_{2}(1-\theta)(1-u)\right)^{2}-\left(1-A \theta \psi_{2}\right)^{2}\right]\left[\left(B \psi_{2}(1-\theta)(1-u)\right) B \psi_{2} \theta u\right. \\
& \left.+\left(1-A \theta \psi_{2}\right) B \psi_{2} \theta u\right]-\left[\left(B \psi_{2}(1-\theta)(1-u)\right)\left(B \psi_{2} u(1-\theta)\right)\right. \\
& \left.+\left(1-A \theta \psi_{2}\right)\left(1+A \psi_{2}-A \theta \psi_{2}\right)\right]\left[\left(B \psi_{2}(1-\theta)(1-u)\right)\left(1+A \psi_{2}-A \theta \psi_{2}\right)\right. \\
& \left.+\left(B \psi_{2} u(1-\theta)+B \psi_{2} \theta(1-u)\right)\left(1-A \theta \psi_{2}\right)\right],
\end{aligned}
$$

respectively.

Due to the complex expressions (31) and (32), conditions $(30)_{5}$ and $(30)_{6}$ are checked partially namely for $\theta=0$ and 1 .

When $\theta=0,(31)$ gives

$$
\begin{aligned}
c_{0}-c_{2} & =\left[\psi_{2}^{2} B^{2}(1-u)^{2}-1\right]^{2}-\left[\left(1+\psi_{2} A\right)\left(\psi_{2} B-\psi_{2} B u\right)+\psi_{2} B u\right]^{2} \\
& +\left[\psi_{2}^{2} B^{2} u(1-u)+\left(1+\psi_{2} A\right)\right]\left[\psi_{2} B\left(1+\psi_{2} A\right)(1-u)+\psi_{2} B u\right], \\
& \geq-\psi_{2}^{2} B^{2} u^{2}+\left[\psi_{2}^{2} B^{2} u(1-u)+\left(1+\psi_{2} A\right)\right] \psi_{2} B u, \\
& =-\psi_{2}^{2} B^{2} u^{2}+\left[\psi_{2}^{2} B^{2} u-\psi_{2}^{2} B^{2} u^{2}+1+\psi_{2} A\right] \psi_{2} B u, \\
& =-\psi_{2} B\left[\psi_{2} B-\left(\psi_{2}^{2} B^{2}-\psi_{2}^{2} B^{2} u\right)-1-\psi_{2} A\right], \\
& >\psi_{2} B-1-\psi_{2} A, \\
& >0, \text { by using } \psi_{2}<\frac{1}{(B-A)}, \text { from (28). }
\end{aligned}
$$


Also when $\theta=1,(31)$ becomes

$$
\begin{aligned}
c_{0}-c_{2} & =\left(1-A \psi_{2}\right)^{4}-\left[\left(B \psi_{2}(1-u)(1-\theta)\right)\right]^{2}-\left[-\left(1-A \psi_{2}\right)^{2}\right]\left[\left(1-A \psi_{2}\right) B \psi_{2} u\right] \\
& +\left(1-A \psi_{2}\right) B \psi_{2}(1-u)\left(1-A \psi_{2}\right), \\
& =\left(1-A \psi_{2}\right)^{4}-\left(B \psi_{2}(1-u)\right)^{2}(1-\theta)^{2}+\left(1-A \psi_{2}\right)^{3} B \psi_{2} u \\
& +\left(1-A \psi_{2}\right)^{2} B \psi_{2}(1-u), \\
& >\left(1-A \psi_{2}\right)^{2}-\left(B \psi_{2}(1-u)\right)^{2}+\left(1-A \psi_{2}\right) B \psi_{2} u+B \psi_{2}(1-u), \\
& >\left(1-A \psi_{2}\right)\left(1-\psi_{2}(A-B)\right)+\left(-B \psi_{2} u+B \psi_{2} u\right)\left(B \psi_{2}-B \psi_{2} u+1\right), \\
& >\left(1-A \psi_{2}\right)\left(1-\psi_{2}(A-B)\right), \\
& >0, \text { by }(28) .
\end{aligned}
$$

If $\theta=0,(32)$ reduces to

$$
\begin{aligned}
c_{0}+c_{2} & =\left[\psi_{2}^{2} B^{2}(1-u)^{2}-1\right]^{2}-\left[\left(1+\psi_{2} A\right)\left(\psi_{2} B-\psi_{2} B u\right)+\psi_{2} B u\right]^{2} \\
& -\left[\psi_{2}^{2} B^{2} u(1-u)+\left(1+\psi_{2} A\right)\right]\left[\psi_{2} B\left(1+\psi_{2} A\right)(1-u)+\psi_{2} B u\right], \\
& >-\psi_{2}^{2} B^{2}-\left[\psi_{2}^{2} B^{2} u(1-u)+\left(1+\psi_{2} A\right)\right] \psi_{2} B, \\
& >-\psi_{2} B\left[\psi_{2} B+\left(\psi_{2}^{2} B^{2}-\psi_{2}^{2} B^{2} u^{2}\right)+\left(1+\psi_{2} A\right)\right], \\
& >\psi_{2} B+1+\psi_{2} A, \\
& >0, \text { by (28). }
\end{aligned}
$$

For $\theta=1$, we have from (32),

$$
\begin{aligned}
c_{0}+c_{2} & =\left(1-A \psi_{2}\right)^{4}-\left[\left(B \psi_{2}(1-u)(1-\theta)\right)\right]^{2}+\left[-\left(1-A \psi_{2}\right)^{2}\right]\left[\left(1-A \psi_{2}\right) B \psi_{2} u\right] \\
& -\left(1-A \psi_{2}\right) B \psi_{2}(1-u)\left(1-A \psi_{2}\right), \\
& =\left(1-A \psi_{2}\right)^{4}-\left(B \psi_{2}(1-u)\right)^{2}(1-\theta)^{2}-\left(1-A \psi_{2}\right)^{3} B \psi_{2} u-\left(1-A \psi_{2}\right)^{2} B \psi_{2}(1-u), \\
& >\left(1-A \psi_{2}\right)^{2}-\left(B \psi_{2}(1-u)\right)^{2}-\left(1-A \psi_{2}\right) B \psi_{2} u-B \psi_{2}(1-u), \\
& >\left(1-A \psi_{2}\right)\left(1-\psi_{2}(A+B)\right)-B \psi_{2}(1-u)\left[B \psi_{2}(1-u)+1\right], \\
& >0 \text { by }(28) .
\end{aligned}
$$

Since all the conditions in (30) are satisfied, all the roots, $\lambda$ of (26) lie within the unit circle for the case under consideration. Therefore the equilibrium point $x^{*}=0$ is asymptotically stable.

Apart from Theorem 5, the case when $B>0$ guarantees the dynamic consistency of our NSFD scheme with respect to positivity as stated in the following result:

Theorem 6. If $B>0$, then the NSFD scheme (25) preserves positivity at all time $t$ whenever the initial conditions are positive.

Proof. Assume that $B>0$ and $x_{0}, x_{1}, \cdots, x_{n} \geq 0$. From (18) and (25) we have, for $m>0$,

$$
\begin{aligned}
x_{n+1} & =\frac{\left[1+A \psi_{2}(\Delta t)-A \theta \psi_{2}(\Delta t)\right] x_{n}+B(1-\theta) \psi_{2}(\Delta t) \tilde{x}_{n}+B \theta \psi_{2}(\Delta t) \tilde{x}_{n+1}}{1-A \theta \psi_{2}(\Delta t)}, \\
& >\frac{\left[1+A \psi_{2}(\Delta t)+B \theta \psi_{2}(\Delta t)\right] x_{n}}{1-A \theta \psi_{2}(\Delta t)}, \text { with } B<-A, \\
& >\frac{\left[1-(-A-B \theta) \psi_{2}(\Delta t)\right] x_{n}}{1-A \theta \psi_{2}(\Delta t)}, \\
& >\frac{1-(-A-B) \psi_{2}(\Delta t)}{1-A \theta \psi_{2}(\Delta t)}, \\
& >0, \text { since } \psi_{2}<\frac{1}{-(A+B)} \text { and } \frac{1}{|A|} \text { by }(28) .
\end{aligned}
$$


Similarly if $m=0$, we have

$$
\begin{aligned}
x_{n+1} & =\frac{\left[1+A \psi_{2}(\Delta t)-A \theta \psi_{2}(\Delta t)+B u \theta \psi_{2}(\Delta t)\right] x_{n}+B(1-\theta) \psi_{2}(\Delta t) \tilde{x}_{n}}{1-A \theta \psi_{2}(\Delta t)-B \theta \psi_{2}(\Delta t)+B u \psi_{2}(\Delta t)}, \\
& >\frac{\left[1+A \psi_{2}(\Delta t)+B \theta \psi_{2}(\Delta t)+B u \theta \psi_{2}(\Delta t)\right] x_{n}}{1-A \theta \psi_{2}(\Delta t)-B \theta \psi_{2}(\Delta t)+B u \psi_{2}(\Delta t)}, \text { with } B<-A, \\
& >\frac{1-(-A-B \theta) \psi_{2}(\Delta t)}{1-(A+B) \theta \psi_{2}(\Delta t)+B \theta u \psi_{2}(\Delta t)}, \\
& >0, \text { since } \psi_{2}<\frac{1}{-(A+B)}, \text { by }(28) .
\end{aligned}
$$

Remark 7: A few comments are in order with respect to the results obtained above. For fixed $\tau$, the case when $m=0$ i.e. $\Delta t>r$ is highly relevant from the nonstandard approach perspective as it allows us to consider arbitrary values of $\Delta t$, a situation which is impermissible in the standard numerical analysis setting. In other words, the impact of the delay on the long term dynamics of the model could be to reduce the step size $\Delta t$ or to increase it.

In view of the analysis done above for $m=0,1,2$ and of the numerical results displayed in Figures 5, 6 and 7, we conjecture that Theorem 5 is valid in the case when $B<0$ and $A>B$ meet the requirements in Theorem 3. Equally, the positivity of the scheme (Theorem 6 ) when $B<0$ is an issue of interest.

\section{Numerical simulations}

In this section, we present numerical simulations that support the theoretical results obtained in the previous sections. As mentioned above and also pointed out in [15], the problem of analysing the location of zeros of a general polynomial such as (26) is a nontrivial problem. This is evident when the delay value $\tau$ or $m$ is large. Moreover, at each value there are different conditions to be satisfied by $\Delta t$. Hence the numerical approach is essential. Here, we show the convergence of solution to the equilibrium point $x^{*}=0$ or the asymptotic stability of this equilibrium using different values of the time step size, $\Delta t$ (different $m$ values), for fixed value of the delay, $\tau, \theta=0,1 / 2$ and different values of $A$ and $B$. In Figure 2 (a) and (b), it has been shown that, starting with initial values close to the fixed-point, delay $\tau=2, A=-0.7$ and $B=-1.3$, the solutions of (25) converge to the fixed-point $x^{*}=0$. Furthermore, the robustness of the NSFD is evident against the Euler scheme and Trapezoidal rule for the same fixed value of the delay. Figure 2 (a), (b) are the NSFD, in which the solutions converge irrespective of $\Delta t$ sizes, in contrast to the Euler scheme and Trapezoidal rule Figure 3 (a) and (b) respectively, which diverges even with much smaller values of $\Delta t$ as indicated. The effect of delay is also apparent in the two schemes: NSFD Figure 2 (c) and Euler Figure 2 (d) without delay, respectively. It should be noticed that models with delay cause the solutions to oscillate before converging to the fixed point, while such phenomenon is absent from models without delay. Moreover, the Euler scheme without delay causes the trajectories to diverge from the fixed point only with slightly higher values of $\Delta t$, compared with the scheme with delay. These facts and simulations regarding models without delay are in agreement with existing results in the literature (see for instance $[2])$. However, the NSFD scheme still converges even with higher values of $\Delta t$.

In Figure 4 (a), the combined Exact and NSFD schemes are shown with $\theta=0$. The exact scheme is defined when $t$ is in $[-\tau, \tau]$. When $t>\tau$, the solution of the linear delay differential equation (4), is shown to be approximated by the NSFD scheme. Figure 4 (b), is the Euler scheme in which the exact nature of our scheme is lost and the poor performance of Euler is observed even with much smaller step size. Figure 4 (c) depicts Theorem 6 , in which the solution is positive at all times when $B>0$ for any positive initial condition. 
From Theorems 4 and 5 , the point $x^{*}=0$ is asymptotically stable fixed-point, for Equation (25) if and only if all the roots of (26) are within a unit circle. This has been shown analytically for some values of $m$ and in Theorem 5 for any value of $m$. For higher values of $m$, this result can be shown numerically. In Figures 5, 6 and 7 with $\theta=0,1 / 2$ and 1, respectively, it can be seen that all the roots of (26) are located within the unit circles for values of $m=0,1,2, \ldots 1000$ (different values of time step sizes, $\Delta t$ ) with fixed delay $\tau=2$ for $B<A<0$.

The Exact-NSFD scheme presented in this paper is primarily designed to handle epidemiological models in a reliable manner. In anticipation to this goal, we consider the delay logistic equation:

$$
\begin{aligned}
x^{\prime}(t) & =B x(t)[1-x(t-\tau)], \quad t>0, B>0, \\
x(t) & =\phi(t)>0, \quad-\tau \leq t \leq 0,
\end{aligned}
$$

which models the transmission dynamics of a wide range of viral diseases such as gonorrhea. For this model, we consider the NSFD scheme

$$
\frac{x_{n+1}-x_{n}}{\psi(\Delta t)}=\left\{\begin{array}{l}
-\frac{B}{\psi(\Delta t)} \int_{t_{n}}^{t_{n+1}} \phi(s-\tau) d s+\frac{\Delta t}{\psi(\Delta t)} B-B\left(x_{n}-1\right)\left[\phi\left(t_{n}-\tau\right)-1\right], \text { if } t_{n+1} \leq \tau, \\
B \phi\left(t_{n}-\tau\right)+B-B\left(x_{n}-1\right)\left[\phi\left(t_{n}-\tau\right)-1\right], \quad \text { if } t_{n} \leq \tau<t_{n+1}, \\
-B(1-\theta) \tilde{x}_{n}-B \theta \tilde{x}_{n+1}+B \\
-B\left[(1-\theta) x_{n}+\theta x_{n+1}-1\right]\left[(1-\theta) \tilde{x}_{n}+\theta \tilde{x}_{n+1}-1\right], \quad \text { if } t_{n}>\tau .
\end{array}\right.
$$

It is clear that the NSFD scheme (34) reduces to the combined Exact-NSFD scheme for the linearized delay logistic equation about the asymptotically stable equilibrium $x^{*}=1$ when we ignore the nonlinear term, $B\left[(1-\theta) x_{n}+\theta x_{n+1}-1\right]\left[(1-\theta) \tilde{x}_{n}+\theta \tilde{x}_{n+1}-1\right]$.

For computation, we use the following explicit expression instead of (34):

$$
x_{n+1}=x_{n}+\left\{\begin{array}{l}
-B \int_{t_{n}}^{t_{n+1}} \phi(s-\tau) d s+\Delta t B-B \psi\left(x_{n}-1\right)\left[\phi\left(t_{n}-\tau\right)-1\right], \quad \text { if } t_{n+1} \leq \tau, \\
B \psi \phi\left(t_{n}-\tau\right)+B \psi-B \psi\left(x_{n}-1\right)\left[\phi\left(t_{n}-\tau\right)-1\right], \quad \text { if } t_{n} \leq \tau<t_{n+1}, \\
-B \psi(1-\theta) \tilde{x}_{n}-B \psi \theta \tilde{x}_{n+1}+B \psi, \quad \text { if } t_{n}>r .
\end{array}\right.
$$

The illustration of the NSFD scheme (34) or (35) is carried out for $\psi_{2}=\frac{\Delta t}{1+(|B| \Delta t)^{2}}, \phi(t)=1+e^{t}$, the set of values $\tau=5.1, B=0.31$ and $A=0$ i.e. $a_{1}=\pi / 2$. In accordance with the dynamics of the delay logistic equations in [6]. Figure 8 shows the NSFD scheme in which the fixed-point $x^{*}=1$ is asymptotically stable for $0<B<\pi / 2 \tau$, irrespective of the step sizes used. The profiles of the discrete solutions confirm that the trapezoidal NSFD scheme $(\theta=1 / 2)$ is more accurate than the Euler scheme $(\theta=0)$. On the contrary, Figure 9 displays the poor performance of both classical Euler and trapezoidal schemes.

\section{Conclusion}

This paper was motivated by the need to construct (reliable) nonstandard finite difference schemes for some epidemiological models defined by delay differential equations. Even for simple models such as the SIR and the SIS - type ones, the presence of delay is a challenge from the numerical point of view. For this reason, and given also the importance of the linearization process in the qualitative 
and constructive analysis of dynamical systems in general and epidemiological models in particular, the focus of the paper changed to the linear delay differential equation. Though being the simplest delay differential equation, designing an exact scheme of this linear model is not an easy task and, in fact, NSFD schemes have not been developed for it.

Using a suitable time splitting, we have in this paper designed a numerical method that combines the advantages of exact and NSFD schemes as follows. At the early time evolution $-\tau \leq t \leq \tau$, where $\tau$ is the value of the delay, we introduce the exact scheme of the linear delay differential equation. Thereafter, we switch to a theta-NSFD scheme constructed by reinforcing Mickens' rules on complex denominator function of the right-hand sides. The later rule is judiciously implemented so that the delay term or the solution at the backtrack point is properly approximated. The main findings are as follows:

(i) The combined Exact-NSFD scheme is dynamically consistent with the linear delay differential equation in many respects.

- It preserves all the properties of the solution at the earlier time evolution.

- It has no spurious fixed-point and it replicates the asymptotic stability property of the trivial equilibrium of the continuous model. These facts are verified both theoretically (under some conditions on the coefficients of the delay differential equation) and computationally when the conditions are not satisfied.

- The profile of the solutions for the combined Exact-NSFD scheme with delay shows oscillations in accordance with the trajectories of the continuous model, while such phenomenon is absent in the same scheme without delay. Furthermore, the better performance (convergence of order 2) of the trapezoidal NSFD scheme is observed.

- When $B>0$ it is shown that the NSFD scheme preserves positivity of solutions at all times irrespective of the step size value $\Delta t$, whenever the initial conditions are positive.

(ii) The robustness of the NSFD scheme is shown in which the fixed point is asymptotically stable irrespective of the large step sizes used, while in the case of classical theta-method, the fixed point is shown to be unstable.

(iii) The relevance of the exact scheme at the early stage of the process is seen in numerical simulations specifically when the delay is longer. On the other hand, for Euler scheme, the delay has effect on the stability of the fixed-point with regards to the smaller step sizes used. For Euler scheme with no delay, the solution profiles converge to the fixed-point for these step sizes while in Euler scheme with delay, the solution profiles diverge for the same smaller step sizes.

In preparation of our future work on general epidemiological models with delay, our theta-NSFD scheme has been adapted for the delay logistic equation. It is seen that the profile of the numerical simulations is in agreement with the dynamics of the underlying continuous model. Other possible areas of application of the NSFD schemes presented here include neuronal synchronization (see for instance $[20,21,22])$.

\section{Acknowledgments}

Three of the authors (SMG, ASH and JM-SL) acknowledge the support of the South African DST/NRF SARChI chair in Mathematical Models and Methods in Bioengineering and Biosciences $\left(M^{3} B^{2}\right)$. ASH acknowledges the support of MacArthur Foundation, Bayero University, Kano, Nigeria. ABG acknowledges, with thanks, the support, in part, of the Natural Science and Engineering Research Council (NSERC) of Canada. The authors are grateful to the anonymous reviewers and Professor T.E. Simos for their constructive comments. 


\section{References}

[1] R. Anguelov, P. Kama, J.M.-S. Lubuma, On non-standard finite difference models of reactiondiffusion equations, Journal of Computational and Applied Mathematics 175 (2005) 11-29.

[2] R. Anguelov and J. M.-S. Lubuma, Contribution to the mathematics of the nonstandard finite difference methods and applications, Numerical Methods Partial Differential Equations, 17 (2001) 518-543.

[3] J. Arino, P. van den Driessche, in: O. Arino et al. (Eds.), Time delays in epidemic models: Modeling and numerical considerations, Springer-Verlag New York, Inc., 2006 pp. 539-578.

[4] C. T. H. Baker, Retarded differential equations, Journal of Computational and Applied Mathematics 125 (2000) 309-335.

[5] R.E. Bellman, K. L. Cooke, Differential-difference equations, Academic Press Inc. London, 1963.

[6] F. Brauer and C. Castillo-Chavez, Mathematical models in population biology and epidemiology, Springer-Verlag New York Inc., 2001.

[7] C. Castillo-Chavez, S. Blower, P. van den Driessche and A. Yakubu, Mathematical approaches for emerging and reemerging infectious diseases: An introduction, Vol. 125, Springer-Verlag New York Inc., 2002.

[8] S. Elaydi, An introduction to difference equations, Springer Science and Business Media Inc. New York, 2005.

[9] J. K. Hale, S. M.V. Lunel, Introduction to functional differential equations, Springer-Verlag, Berlin, Heidelrberg, New York, 1993.

[10] H. W. Hethcote, P. van den Driessche, An SIS epidemic model with variable population size and a delay, Journal of Mathematical Biology, 342 (1995) 177-194.

[11] H. W. Hethcote, P. van den Driessche, Two SIS epidemiologic models with delays, Journal of Mathematical Biology, 40 (2000) 3-26.

[12] A.R. Humphries, O.A. DeMasi, F.M.G. Magpantay and F. Upham, Dynamics of a delay differential equation with multiple state dependent delays, Discrete and Continuous Dynamical Systems series A, 32 (2012) 2701-2727.

[13] E. I. Jury, Theory and applications of the $\mathrm{z}$ - transform method, Robert E. Krieger Publishing Co., Huntington, New York, 1964.

[14] J. M.-S. Lubuma and A. Roux, An improved theta-method for systems of ordinary differential equations, Journal of Difference Equations and Applications, 911 (2003) 1023-1035.

[15] T. Luzyanina, D. Rose, Numerical stability analysis and computation of hopf bifurcation points for delay differential equations, Journal of Computational and Applied Mathematics, 72 (1996) 379-392.

[16] R. E. Mickens, Applications of nonstandard finite difference schemes, World Scientific Publishing Co. Pte. Ltd., 2000.

[17] R. E. Mickens, Nonstandard finite difference models of differential equations, World Scientific, 1994.

[18] G. A. Ngwa, A. M. Niger and A. B. Gumel, Mathematical assessment of the role of non-linear birth and maturation delay in the population dynamics of the malaria vector, Applied Mathematics and Computation 217 (2010) 3286-3313. 
[19] M. A. Safi, A. B. Gumel, The effect of incidence function on the dynamics of a quarantine/isolation model with time delay, Nonlinear Analysis: Real World Applications 12 (2011), $215-235$.

[20] Q. Wang, G. Chen and M. Perc, Synchronous Bursts on Scale-Free Neuronal Networks with Attractive and Repulsive Coupling. PLoS ONE 6(1) (2011) e15851. doi:10.1371/journal.pone.0015851.

[21] Q. Wang, M. Perc, Z. Duan and G. Chen, Synchronization transitions on scale-free neuronal networks due to finite information transmission delays, Phys. Rev. (2009) E 80, 026206.

[22] Q. Wang, Z. Duan, M. Perc and G. Chen, Synchronization transitions on small-world neuronal networks: Effects of information transmission delay and rewiring probability, (2008) EPL 83 50008 doi:10.1209/0295-5075/83/50008.

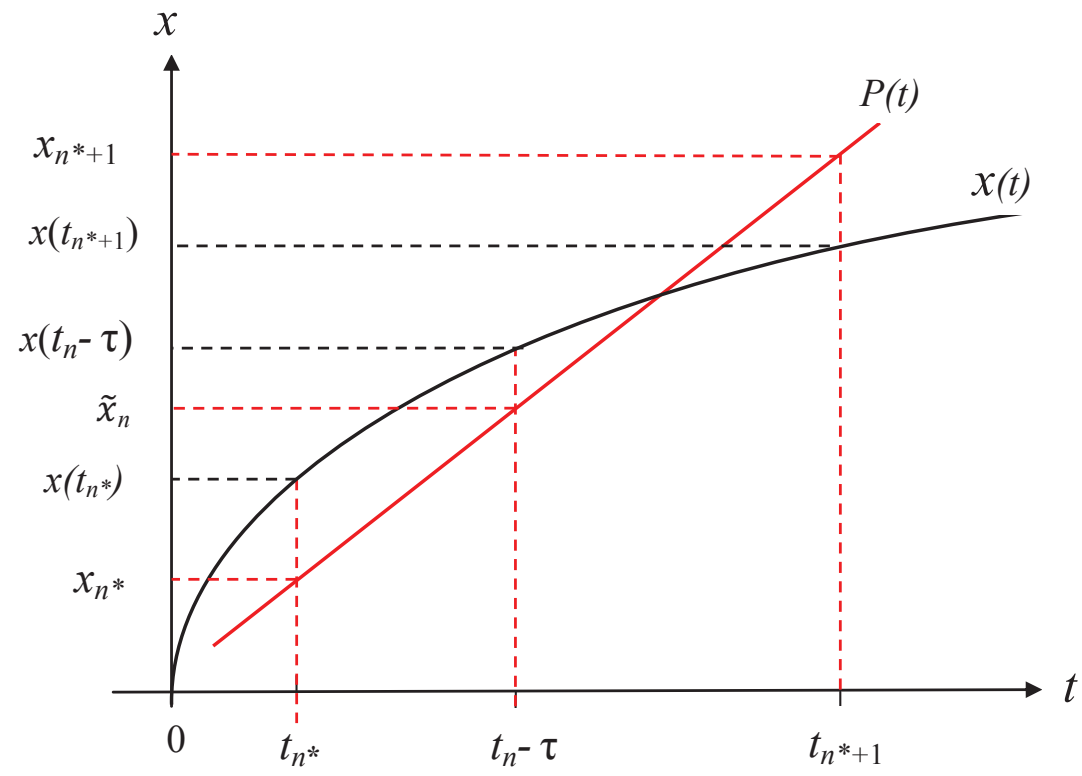

Figure 1: Approximation of the delay term $x\left(t_{n}-\tau\right)$. 

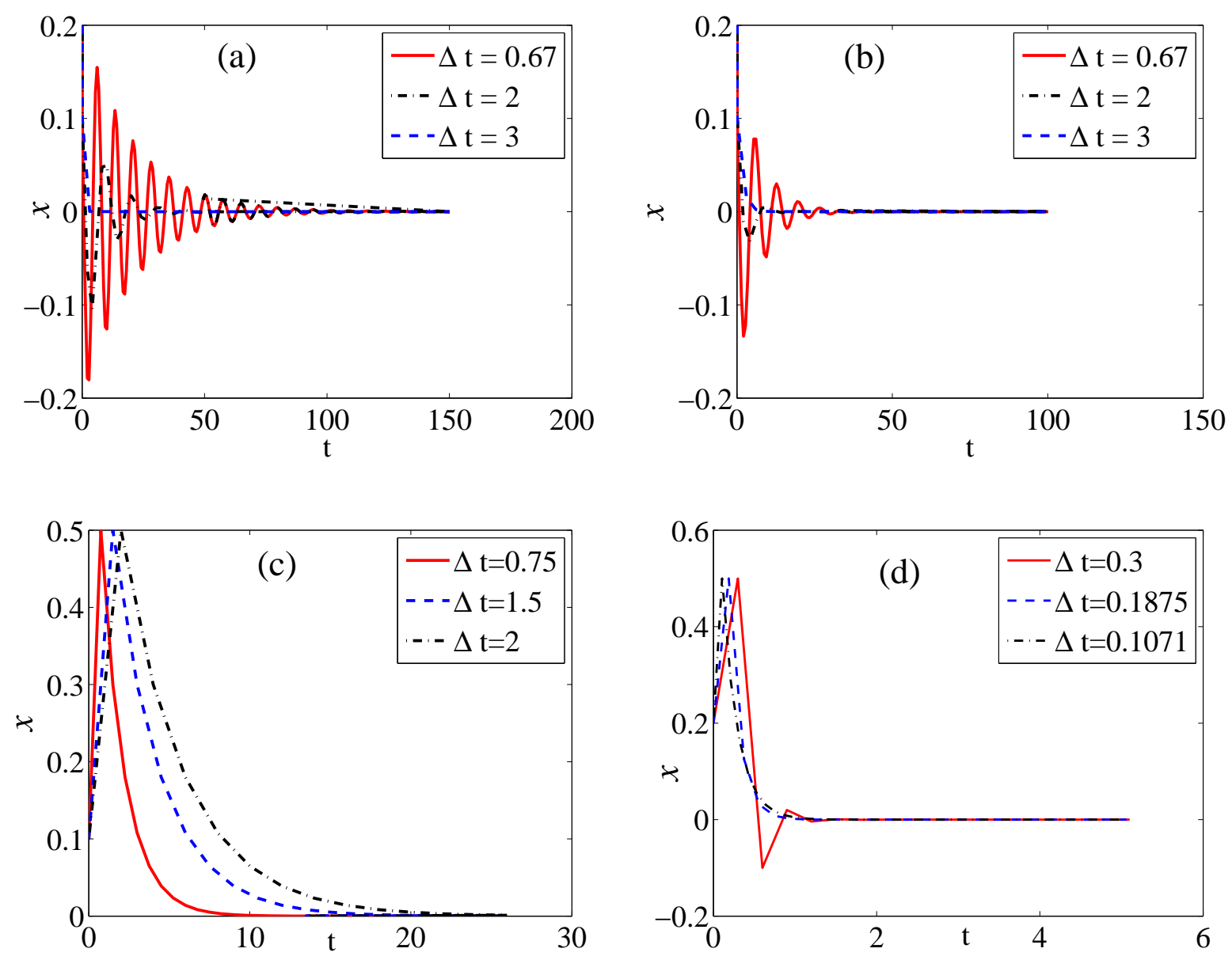

Figure 2: For $A=-0.7, B=-1.3$ NSFD schemes with $\tau=2$, (a) $\theta=0$ and (b) $\theta=1 / 2$, while in (c) NSFD and (d) Euler schemes without delay.
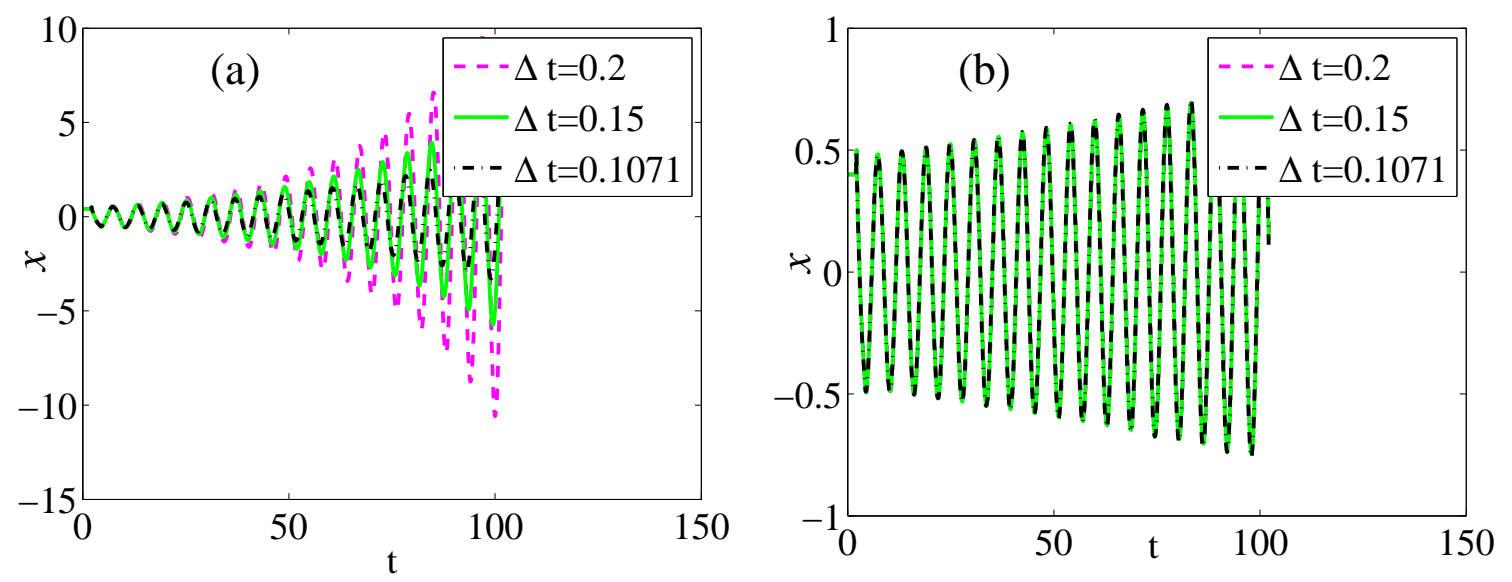

Figure 3: For $A=-0.7, B=-1.3$ and $\tau=2$ : (a) Euler scheme and (b) Trapezoidal rule. 

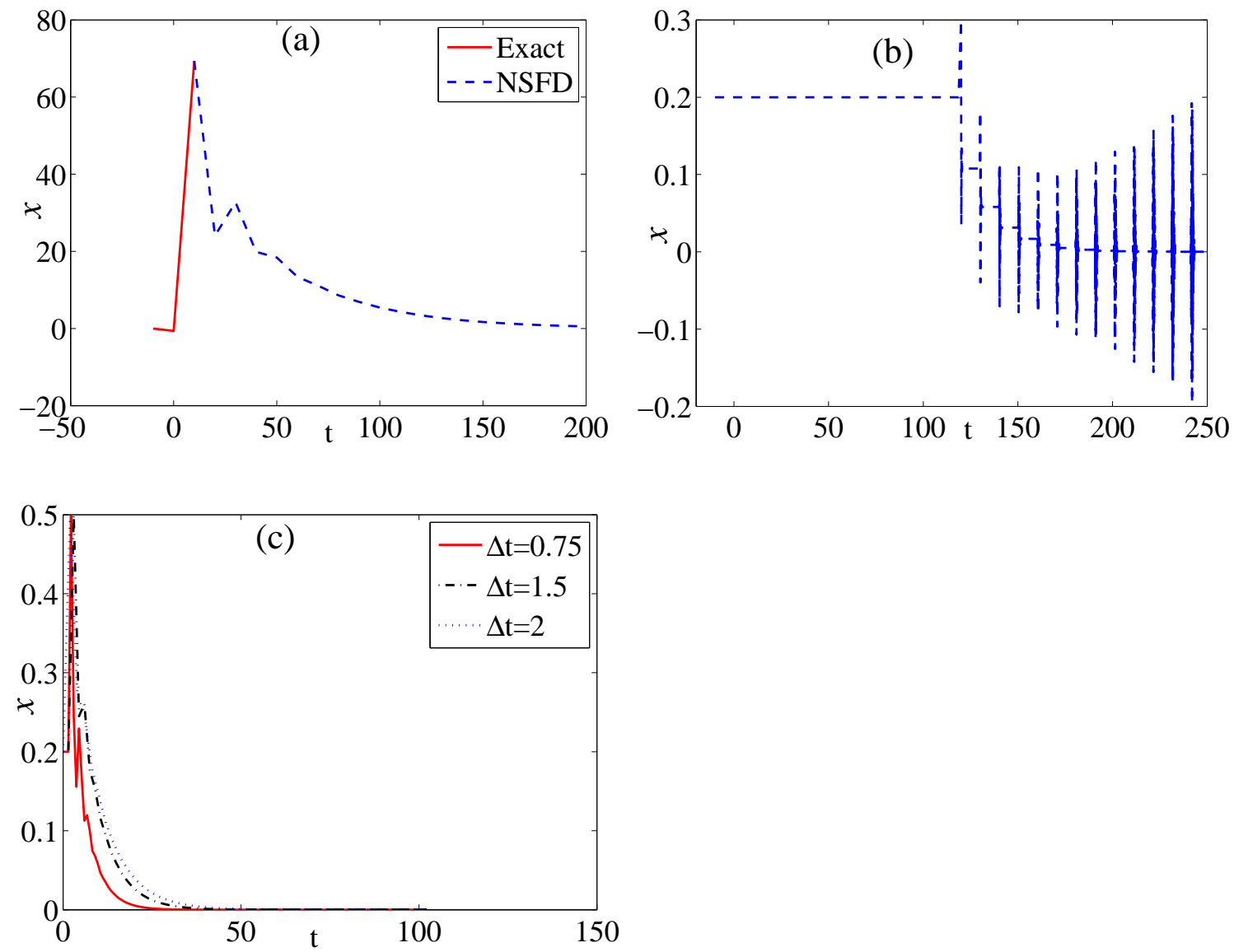

Figure 4: For $A=-13, B=7, \tau=10$; (a) Combined Exact-NSFD scheme, $\Delta t=10$, $\theta=0$ (b) Euler scheme, $\Delta t=0.11$ (c) Positivity of solution (Theorem 6). 

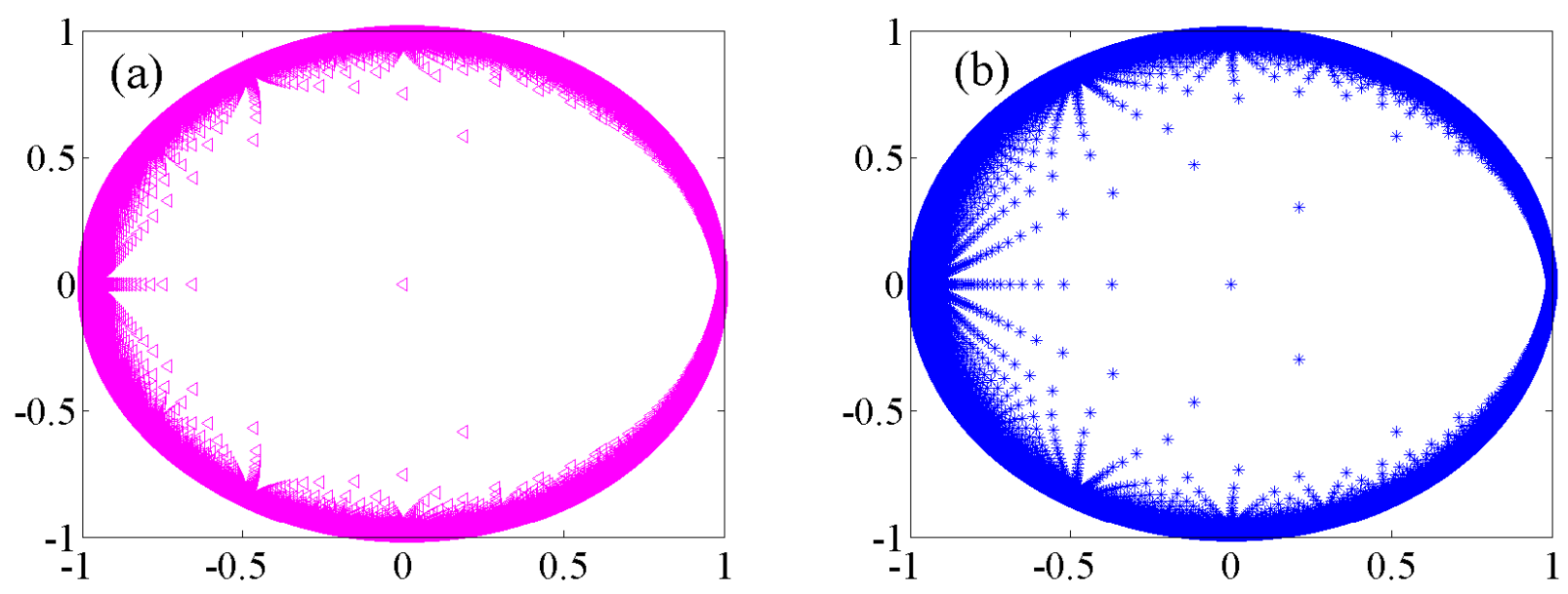

Figure 5: The roots of the characteristic polynomial for (26) within unit circles corresponding to values of $m=0,1,2, \ldots 1000$ (different values of $\Delta t$ ), $\tau=2, A=-1.3$, $B=-1.7$ in (a) $\theta=0$, (b) $\theta=1 / 2$.
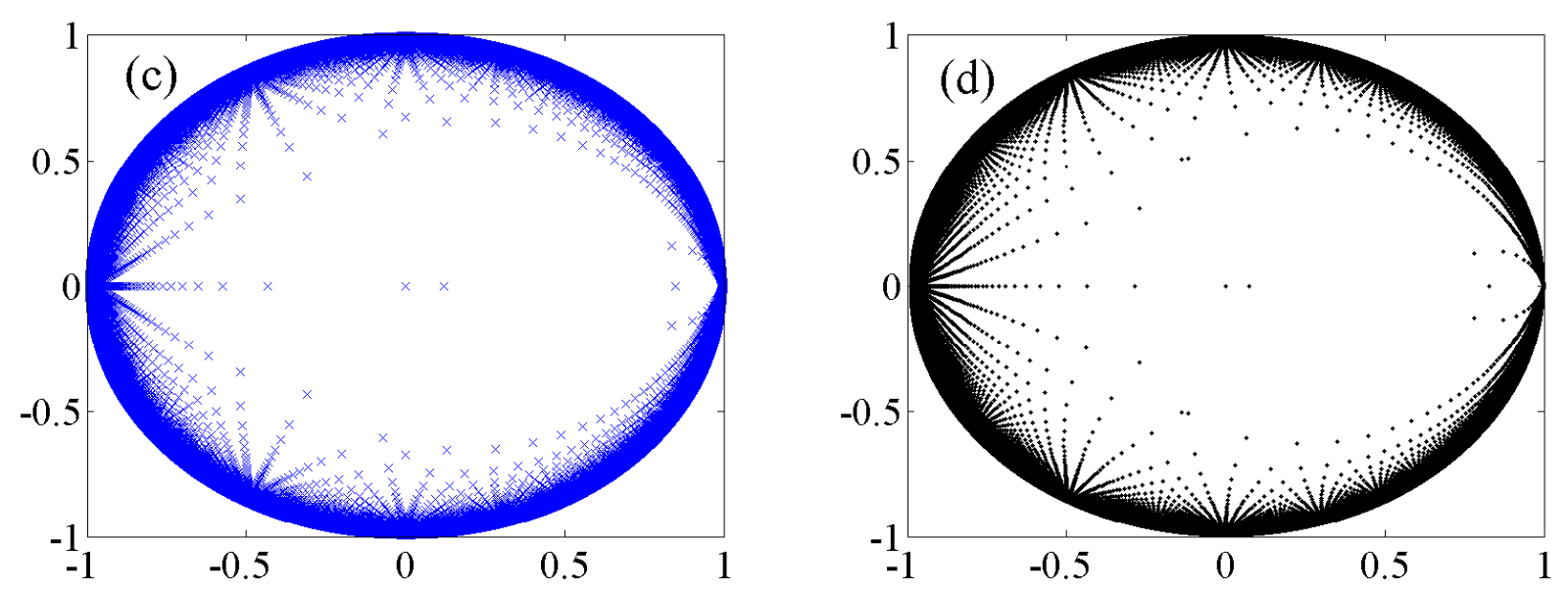

Figure 6: The roots of the characteristic polynomial for (26) within unit circles corresponding to values of $m=0,1,2, \ldots 1000$ (different values of $\Delta t$ ), $\tau=0.54, A=1.3$, $B=-1.7$ in $(\mathrm{c}) \theta=0$, (d) $\theta=1 / 2$. 

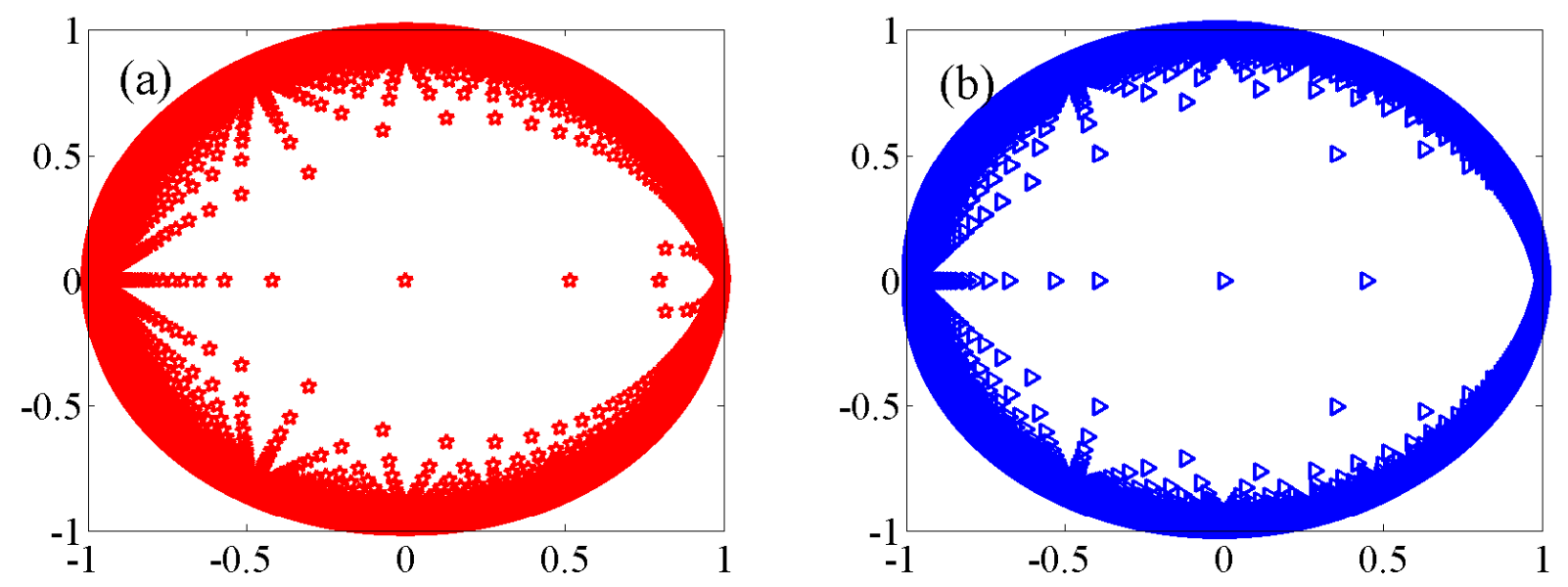

Figure 7: The roots of the characteristic polynomial for (26) within unit circles corresponding to values of $m=0,1,2, \ldots 1000$ (different values of $\Delta t$ ), $\theta=1$, in (a) $\tau=0.54, A=1.3, B=-1.7$ in (b) $\tau=2, A=-1.3, B=-1.7$.
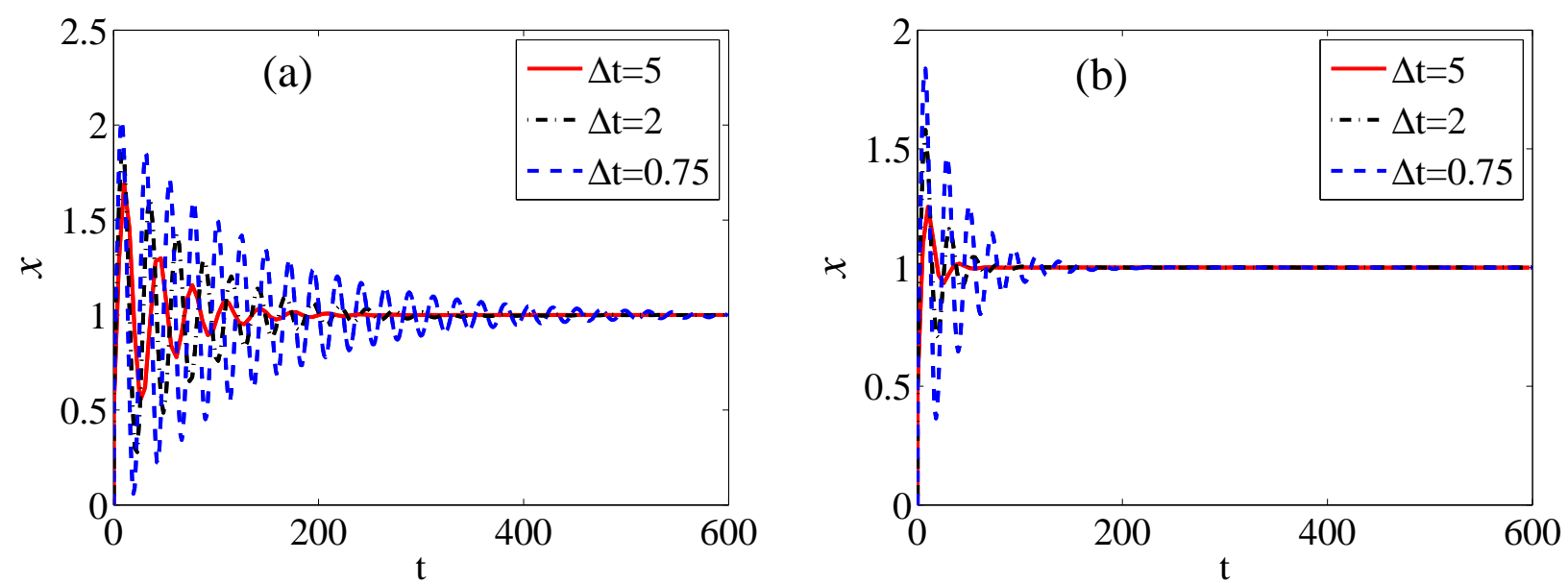

Figure 8: NSFD schemes for $\tau=5.1, B=0.31$; in (a) $\theta=0$ (b) $\theta=1 / 2$. 

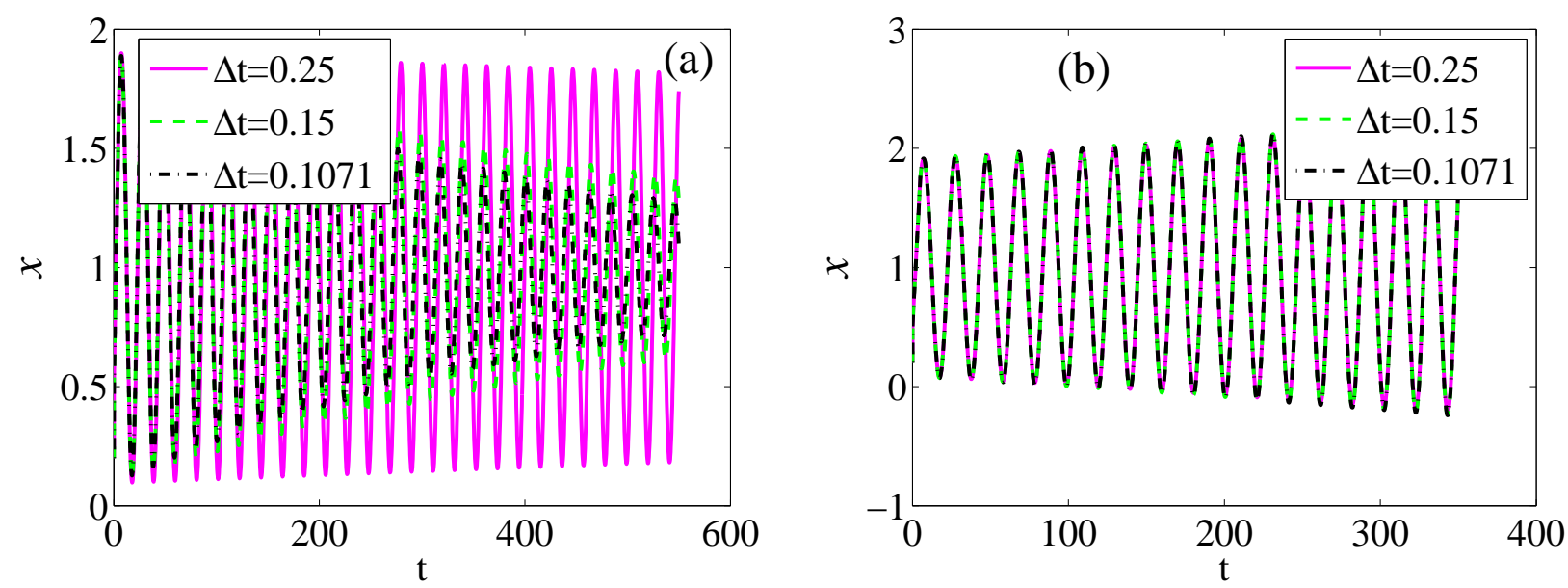

Figure 9: For $\tau=5.1$ and $B=0.31$ : (a) the Euler scheme (b) Trapezoidal rule. 